\title{
Experimental Evaluation of the Age of Information via Emulation
}

\author{
Clement Kam*, Sastry Kompella*, and Anthony Ephremides ${ }^{\dagger}$ \\ *Information Technology Division, Naval Research Laboratory, Washington, DC 20375 \\ ${ }^{\dagger}$ Electrical and Computer Engineering Department, University of Maryland, College Park, MD 20742
}

\begin{abstract}
This work focuses on evaluating a new metric for the timeliness of information in a status monitoring system, referred to as the age of information or the status age. Investigation into the age of information metric is fairly recent and has primarily been focused on theoretical analysis, and there has been no evaluation of the metric in a realistic system of networked nodes. We evaluate the age metric in a realistic wireless system using the open source network emulation tools CORE and EMANE. Our goal is to validate theoretical results on age for a single link across a wide range of system parameters. In addition to verifying existing theoretical results, we go further by adding more theoretical results that better model the emulated system than currently offered in the theory. Our results indicate that the theoretical results are indicative of real world behavior, but we also identify the limitations of the theoretical models and the impact on the emulated age.
\end{abstract}

\section{INTRODUCTION}

There is a category of applications that can be described as status monitoring, in which some content or piece of information is regularly updated and transmitted to a monitor, and the application is only interested in the latest status for this content. Some examples include tracking position/location, monitoring vital signs, and surveillance. For such applications, it is not crucial to have all of the information about the status for all time, but instead only the most recent information is of interest. Therefore, the timeliness or "freshness" of information is the most relevant measure for these systems.

Traditional network metrics of throughput and delay are inadequate for describing the performance of these status monitoring applications. For example, the delay for a particular packet may be short, but if its transmission occurred a long time ago, the information, as observed at the current time, is no longer fresh. As another example, throughput may be high, such that packets arrive very frequently at the receiver. However, if the packets that arrive were generated a long time ago but delayed in a queue (at the source node or a relay node), even recently received packets are no longer fresh. A different metric is needed to convey the freshness of information at the receiver.

Recently, a new metric known as the age of information or status age was introduced by Kaul et al. in the context of vehicular networks [1], [2]. They continued this work by characterizing the status age for a system consisting of a singleserver queue with a first-come-first-served discipline [3]. It was shown that when packets are generated infrequently, the monitor receives packets infrequently, and average status age can be large, i.e., what the monitor observes, on average, is an old status. Increasing the rate of packet generation (or sampling) initially reduces the age. However, at some point, packets are generated too frequently, which backlogs the queue; consequently, packets spend a long time in the queue before reaching the monitor. As a result, there is an optimal rate at which packets can be generated to minimize the average age. In [4], a single server with last-come-first-served queue discipline was studied, and it was shown that increasing the utilization will always reduce the average age since the newest packets are sent first and older packets have no effect on the age. Additional work on status age focused on systems with multiple sources [5] and the effect of packet management [6].

The age of information was later brought into the network setting, modeling the network as a simple system with infinite packet capacity and random delay to the destination [7]. The network was modeled as an $\mathrm{M} / \mathrm{M} / \infty$ queue, in which packets do not undergo any queuing delay, but packets can arrive out of order. This work was then extended to an intermediate case between a single server queue and an infinite server system to an $\mathrm{M} / \mathrm{M} / 2$ queue, which experiences both queuing delay and out-of-order arrival [8].

In this work, we focus on validating some of the existing theoretical studies on the age of information over a single link via network emulation. We conduct emulation tests in which we test over a wide range of parameters such as packet arrival rate, arrival distribution, numbers of flows, and packet loss. Our emulation results demonstrate that the theoretical results are indicative of realistic network performance. We also introduce some new theoretical results and highlight some limitations of the theoretical analysis.

\section{Age OF INFORMation}

We are interested in testing the age of information metric for status updating applications. For example, we consider the system of a first-come, first-served queue in FIgure 1. The age metric is defined as $\Delta(t)=t-u(t)$, where $u(t)$ is the time stamp of the most recently received packet by the monitor. Following the example shown in Figure 2, a packet is received at the monitor at time $\tau_{0}$, and at time 0 , the age is equal to $-t_{0}$. As time progresses, the information ages linearly until the next packet is received at time $\tau_{1}$, which has an age equal to $\tau_{1}-t_{1}$. Continuing this pattern, we observe that the function 


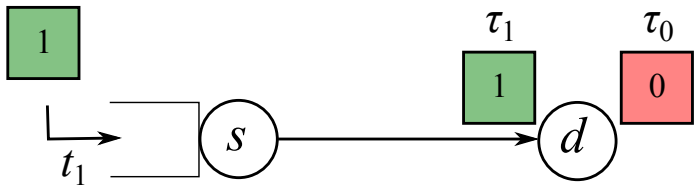

Fig. 1. First-come, first-served queue.

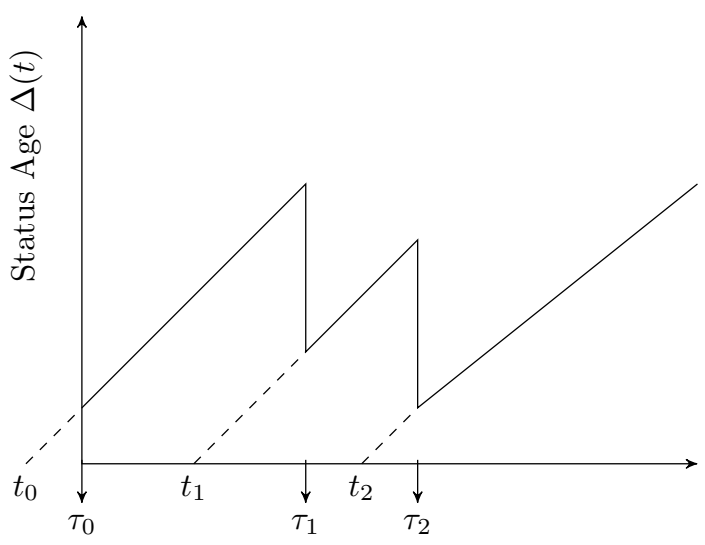

Fig. 2. Age of information plot.

exhibits a "sawtooth" pattern that is characteristic of the age function.

In the theoretical studies on the age, the typical goal is to analyze the average performance of the age as a function of the packet generation rate. The time-average age is computed using a graphical approach to compute the area under the sawtooth curve, specifically by computing the average area of a trapezoidal shape associated with each packet updating the age. This is a non-trivial computation even for simple systems (e.g., M/M/1 queue), since it involves computing the expected value of the product of the packet interarrival time at the source and the system time, which are not independent.

As an alternative to the average age, the peak age, which observes the peak values of the sawtooth function, was proposed in [6]. It provides information on the worst case age (on average over all packets), and is easy to calculate compared to the average age. The average peak age is given by

$$
E\left[A_{k}\right]=E\left[X_{k}\right]+E\left[T_{k}\right] .
$$

Even for slightly more complex networks, the average age analysis would be very difficult, and simulation would be a useful approach for evaluating the age performance. However, simulation may not account for the effects of real systems, such as processing time, network interface queuing delays, finite buffers, among other considerations. Before venturing into more complex networks, we conduct this study as a way to (approximately) validate the theoretical results, while investigating the impact of real system-level effects on the age of information metric.

\section{EMUlation TOOLS}

Thus far, the work on the age of information has focused primarily on theoretical studies with idealized models and mathematical analysis. This study focuses on evaluating the age metric in a realistic network emulation testbed, thus serving as a necessary supplement to the theoretical studies. Our aim is to begin work on studying the age metric by considering a single link and testing across a variety of parameters that arise in real systems. This serves as a foundation for studying the age metric in larger, more complex networks, for which mathematical analysis of the age metric is a difficult open problem.

We build our emulation testbed using the Common Open Research Emulator (CORE) [9] and the Extendable Mobile Ad-hoc Network Emulator (EMANE) [10], which are tools developed in part by the Naval Research Laboratory (NRL). We use CORE and EMANE in conjunction as in [11], where CORE is used to emulate layers 3 and above of the protocol stack (network, transport, session, application), and EMANE serves as a high fidelity layer 1 and 2 emulator (physical and data link).

These tools enable the creation of virtual networks consisting of lightweight virtual machines as the emulated nodes. This is a cost-effective approach to realistic network performance evaluation, in which the actual Linux network stack is used with user applications, and wireless channel effects can be emulated in real-time. A wide variety of system configurations are possible with CORE and EMANE's pre-packaged models and services, including heterogenous wired/wireless networks, routing protocols, alternate MAC/PHY models, among others.

\section{Testbed Setur}

The system used for testing was a MacBook Pro notebook computer with a dual-core $3 \mathrm{GHz}$ Intel Core i7 (Turbo Boost up to $3.5 \mathrm{GHz}$ ) CPU with $256 \mathrm{~KB} \mathrm{L2}$ and $4 \mathrm{MB} \mathrm{L} 3$ caches, and $16 \mathrm{~GB}$ of $1600 \mathrm{MHz}$ DDR3 RAM and a $250 \mathrm{~GB}$ solid state disk. This notebook was running Mac OS X Yosemite (10.10). The CORE/EMANE environment was run on a virtual machine using VirtualBox 4.3.26 to run 64-bit Ubuntu 12.04.5 LTS on a 3.13.0-46-generic Linux kernel. The CORE 4.7 and EMANE 0.9.1 versions were installed.

We use a simple 2 node setup shown in Figure 3. Static routes are used, so there is no routing overhead. We use EMANE's RF-PIPE model, which is a generic radio effects MAC model coupled with EMANE's Universal PHY layer. The RF-PIPE MAC uses the SINR to compute a packet loss probability, and the user can configure a channel data rate, delay, and jitter. For all experiments, we use a data rate of 1 Mbps, and we generate 1000 byte packets, including overhead. This yields a maximum packet rate of 125 packets per second over the channel.

Traffic is generated using NRL's multi-generator (MGEN) network test tool, which tests IP network performance using TCP and/or UDP/IP traffic. MGEN logs the packet generation time and the packet reception time. We created tools to trace through MGEN log files to generate packet latency data files for each flow, which record the latency and reception time alongside the packet ID. From these latency files, we generate 


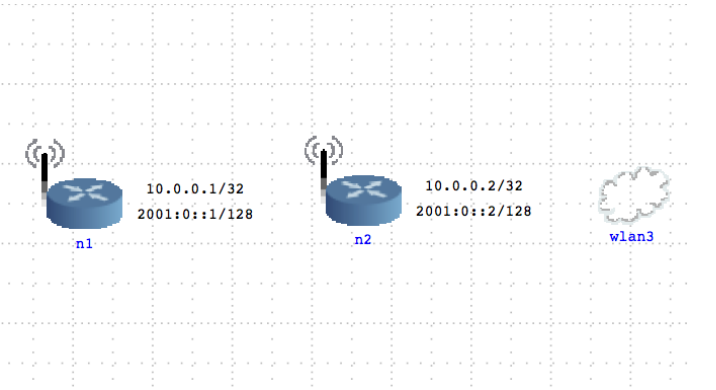

Fig. 3. Screenshot of CORE GUI: two node wireless link.

files that record the packet age as a function of time as well as the average age and average peak age for each test run. Each test is run for 30 seconds (warmup) before recording the data used in our results. After the warmup, the test is run such that, on average, 20 packets are transmitted, but the run can be no less than 10 seconds. We average our results over 50 runs. The packet rates presented are for rates less than the system service rate, which is the typical operating regime, beyond which leads to queue overload and unpredictable latency. Since the latency is dependent on a number of queues in the system, including the specific implementation of the emulated model, we do not seek to understand the inner workings of our particular system and ignore this operating regime in this work.

\section{THEORY VS. EMULATION}

In this section, we compare the age performance in emulation with the theoretical performance. For our emulation setup, we use EMANE's RF-PIPE MAC/PHY model with a delay and jitter set to 0.1 seconds. This is equivalent to a random packet service time with a uniform distribution between 0 and 0.2 seconds $(U[0,0.2])$. Figure 4 shows a snippet of the characteristic sawtooth age function for a generation rate of 2 packets per second (Poisson distributed), plotted along with the packet generation time and the latency. With an average delay set to 0.1 seconds, the packet queues begin to saturate at a rate of about 10 packets per second. Figure 5 shows a snippet for a generation rate of 9.5 packets per second, and we notice that the latency closely follows the age function. Note that we are well below the maximum packet rate over the channel of 125 packets per second.

Some of the first results in the theory were derived for the $\mathrm{M} / \mathrm{M} / 1$ queue, and we are interested in how well it models different arrival and departure processes in our network emulation, such as the delay/jitter departure process in RF-PIPE. EMANE does not have a model that has an exponentially distributed service time, and the delay/jitter model has a simple mathematical model. On the other hand, there have been no theoretical results on the age that have considered the delay/jitter departure process used here. Therefore, we provide theoretical results based on the delay/jitter model to compare with the emulation results.

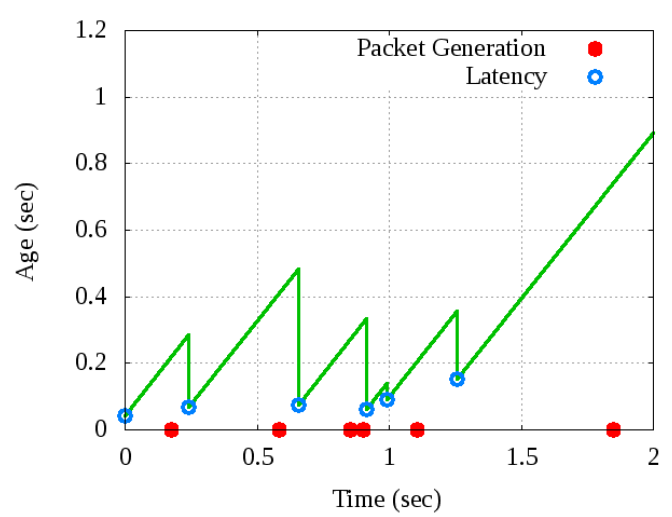

Fig. 4. Age and latency, 2 packets/second.

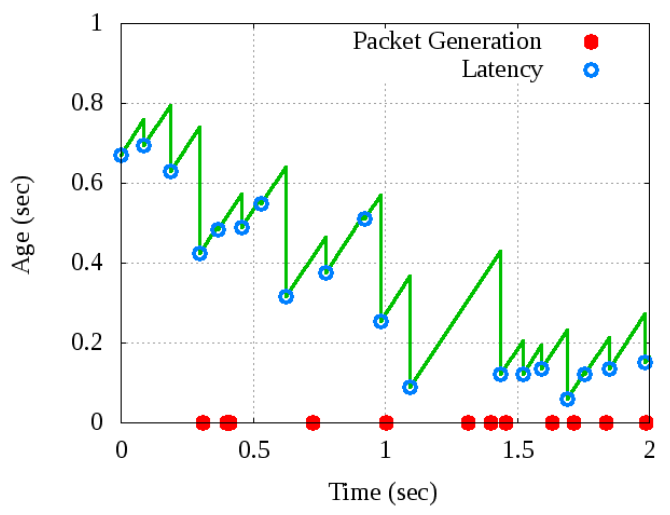

Fig. 5. Age and latency, 9.5 packets/second.

\section{A. Average Age for an $M / M / 1$ Queue}

We begin our investigation with a system in which there is a single flow of packets for which we would like to compute the average age. Due to the difficulty of computing the age for the uniform service time distribution (delay/jitter) used in our emulation, we compare our results to the theoretical results for an exponential service time model, or the $M / M / 1$ [3]. The average age for an $\mathrm{M} / \mathrm{M} / 1$ queue is given by

$$
\Delta=\frac{1}{\mu}\left(1+\frac{\mu}{\lambda}+\frac{\lambda^{2}}{\mu^{2}-\lambda \mu}\right)
$$

Figure 6 plots the average age alongside the average interarrival time of packets as well as the average packet latency, and these per-run averages are averaged over 50 runs. We include $1 \sigma$ error bars here and in the remaining plots in this work. We are interested in comparing the age performance to other performance metrics (i.e., delay and rate). We first consider the average interarrival time. As the interarrival rate $\lambda$ approaches zero, the average age approaches $1 / \lambda$, which is equal to the interarrival time. However, the age eventually starts to increase as $\lambda$ approached $\mu$, whereas the interarrival time approaches zero. This is verified in our emulation results shown in Figure 6.

Turning our attention to the average packet latency, we first note that in real systems, packet queues have a finite capacity, 


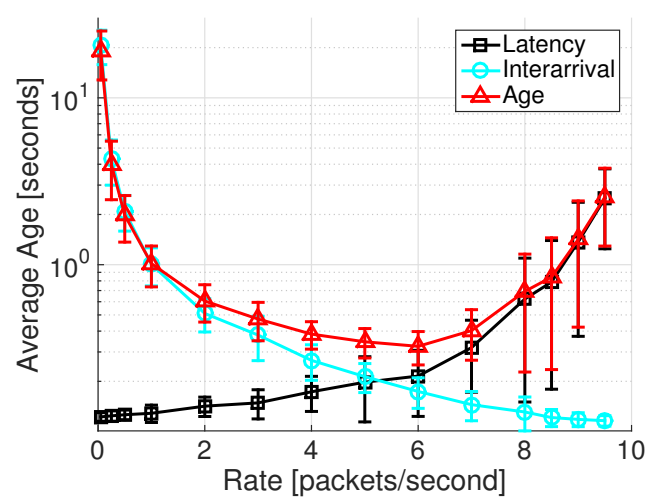

Fig. 6. Emulated average age/latency/interarrival, $1 \sigma$ error bars.

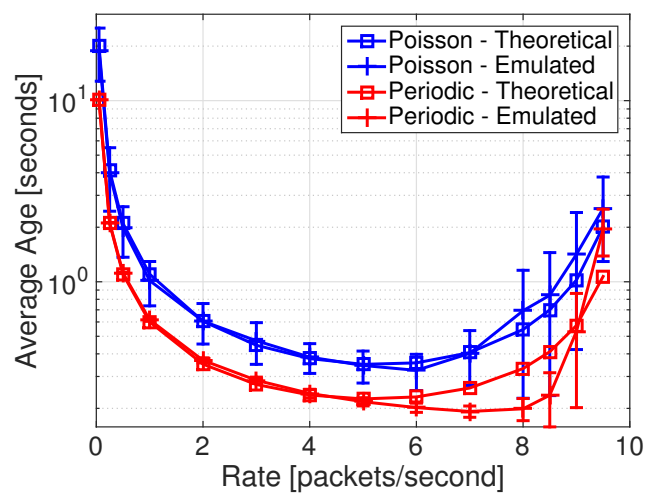

Fig. 7. Average age, comparing Poisson and periodic interarrivals. whereas the M/M/1 assumes an infinite queue capacity. The theoretical work on age has not yet considered finite capacity queues due to the analytical difficulty. For the finite queue (in the emulation) without any queue management, packets that are generated when the queue is full are dropped. We consider the general form of the age of information in terms of the average packet latency, which is the time between the generation of the packet to the time of its arrival at the receiver. This is also known as the system time $T$, and the age of information for a general first-come, first-served system is given by

$$
\Delta=\lambda\left(E[X T]+E\left[X^{2}\right] / 2\right) .
$$

As $\lambda$ approaches zero, the system time (or packet latency) approaches the average service time ( 0.1 seconds in our case), since the queue is nearly always empty. For large $\lambda$ as it approaches or exceeds $\mu$, we approximate that $X$ and $T$ are independent. The justification is that in such cases, the queue is full almost all the time, and thus the system time $T$ only depends on the time required for a full queue to be served. With this approximation, we have

$$
\begin{aligned}
\Delta & =\frac{1}{E[X]}\left(E[X T]+E\left[X^{2}\right] / 2\right) \\
& \approx \frac{E\left[X^{2}\right]}{2 E[X]}+E[T] \\
& =\frac{1}{\lambda}+E T
\end{aligned}
$$

which approaches $E[T]$, the average system time, for large $\lambda$. Thus, the average age and the average packet latency are approximately the same for large $\lambda$ and $\lambda$ approaches or is greater than $\mu$. This is what we observe in Figure 6.

\section{B. Average Age for a D/M/l Queue}

Next, we consider the impact of a different arrival process on the age performance. For a periodic packet arrival process (D/M/1 queue) with period $D[3]$, the packet arrival rate is

$$
\Delta=\frac{1}{\mu}\left[\frac{1}{2 \rho}+\frac{1}{1-\beta}\right]
$$

where

$$
\beta=-\rho \mathcal{W}\left(-\rho^{-1} e^{(-1 / \rho)}\right)
$$

and $\mathcal{W}(\cdot)$ is the Lambert $\mathrm{W}$ function, and the utilization $\rho=$ $1 /(\mu D)$.

In Figure 7, we compare the average age for Poisson and periodic arrival processes from the theoretical computations as well as from emulation results. We see that the periodic age yields a slightly lower average age than the Poisson, which was noted in the theoretical analysis [3]. We also note that as the rate approaches 10 packets/second (i.e., toward queue saturation), the emulation performance slightly diverges from the theory, due to the unpredictability of the real system queueing behavior. In particular, the average age for the periodic case continues to decrease until 7 packets/second, which is beyond what is predicted in theory. This may be due in part to the service time being uniformly distributed, whereas the theoretical result is based on an exponential distribution.

To explain this discrepancy, we consider a system in which the arrival process is periodic and service time is deterministic (i.e., a $\mathrm{D} / \mathrm{D} / 1$ queue). For such a system, the age will continue to decrease until the arrival rate matches the service rate $(\lambda=$ $\mu)$. Since the service time here is modeled as $U[0,0.2]$, it has some characteristics of the deterministic service time, in that it cannot be greater than 0.2 seconds. Therefore, one would expect the age to reach its minimum at a higher rate than that of the exponentially distributed service time model (but not all the way at $\lambda=\mu$ as in the deterministic case). This effect is not observed when the arrivals are Poisson since there is no matching between the regularity of the arrival and service processes.

\section{Peak Age for an $M / G / 1$ Queue}

We now consider computing the age for the exact service time distribution used in emulation, which is $U[0,0.2]$. Due to the difficulty of computing the average age, we only consider the peak age in this case, given by (1). By the PollaczekKhinchin formula, the average waiting time for an $\mathrm{M} / \mathrm{G} / 1$ 


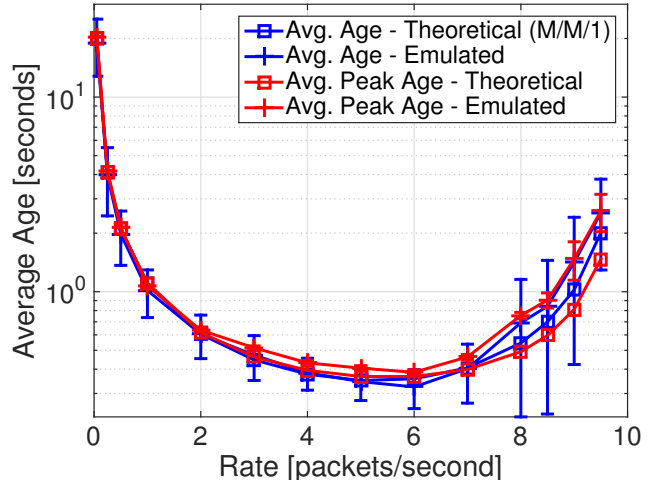

Fig. 8. Comparing theoretical and emulated average peak age, Poisson interarrivals.

where the service time is $U[0,2 / \mu]$ is given by

$$
\begin{aligned}
W & =\frac{\lambda \overline{X^{2}}}{2(1-\rho)} \\
& =\frac{2 \lambda}{3 \mu(\mu-\lambda)} .
\end{aligned}
$$

Thus the average peak age is

$$
E\left[A_{k}\right]=\frac{1}{\lambda}+\frac{1}{\mu}+\frac{2 \lambda}{3 \mu(\mu-\lambda)} .
$$

Figure 8 shows results for the average age and average peak age for Poisson arrivals. For the average age, we used the results for the M/M/1 model. The theory and emulation results match up well, and for smaller rates, the average age and average peak age are very similar, which can be seen from comparing (5) with (2).

We also have plotted the average age and average peak age for a periodic arrival process in Figure 9. In this case, we compared the emulation results with the theoretical age for a $\mathrm{D} / \mathrm{M} / 1$. Using the expression for $E[T]$ in [3], we compute the average peak age for a $\mathrm{D} / \mathrm{M} / 1$ system:

$$
E\left[A_{k}\right]=\frac{1}{\lambda}+\frac{1}{\mu}+\frac{\beta}{\mu(1-\beta)} .
$$

Unlike the case of Poisson arrivals (Figure 8), the difference between the average peak age (6) and the average age (4) is quite apparent and is confirmed in the results in Figure 9.

\section{Two Flows}

We now consider the age performance for a system with multiple flows. Specifically, we look at two flows being sent from node 1 to node 2 . The age for multiple sources was studied in [5], and the average age for source 1 in a two source system with rates $\lambda_{1}$ and $\lambda_{2}$ entering an M/M/1 queue was shown to be

$$
\Delta_{1}=\frac{1}{\mu}\left[\frac{\rho_{1}^{2}\left(1-\rho \rho_{2}\right)}{(1-\rho)\left(1-\rho_{2}\right)^{3}}+\frac{1}{1-\rho_{2}}+\frac{\rho_{2}}{\rho_{1}\left(1-\rho_{2}\right)}\right]
$$

where $\rho_{1}=\lambda_{1} / \mu, \rho_{2}=\lambda_{2} / \mu$, and $\rho=\rho_{1}+\rho_{2}$. The expression for $\Delta_{2}$ is given by swapping the subscripts for $\rho_{1}$

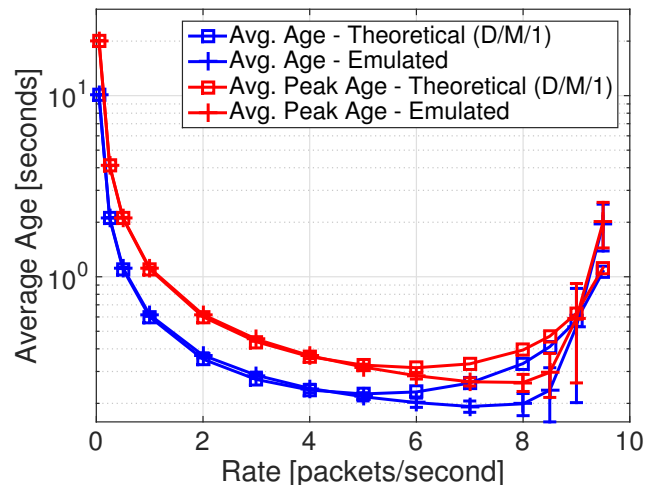

Fig. 9. Comparing theoretical and emulated average peak age, periodic interarrivals.

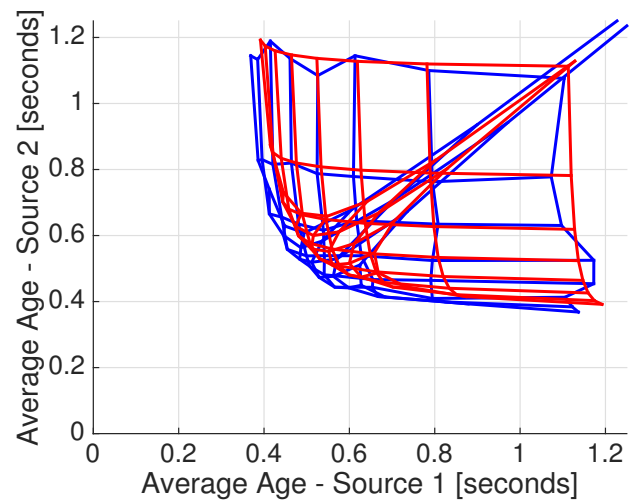

Fig. 10. Average age for two sources, theoretical (red) vs. emulated (blue).

and $\rho_{2}$. We have plotted the theoretical and emulated results in Figure 10. The age is minimized when $\rho_{1}=\rho_{2} \approx 0.3$, which confirms the findings in [5].

\section{E. Impact of Packet Loss}

Thus far, the theoretical work on the age of information has not considered the impact of packet losses on the age metric. Considering an $\mathrm{M} / \mathrm{M} / 1$ system, analyzing $E[X T]$ presents some challenges due to $X$ depending on packet losses while $T$ does not, so we resort to analyzing the peak age, given in (1). Given that the probability $p_{s}$ of each packet being successful is independent, the interarrival process of packets that are not lost is known as a thinned Poisson process, which is also Poisson with rate $p_{s} \lambda$. The value of $E[T]$ is computed according to a Poisson arrival with rate $\lambda$ and $E[X]$ is computed according to the thinned arrival, yielding an average peak age of

$$
E\left[A_{k}\right]=\frac{1}{\mu}\left(\frac{1}{1-\rho}+\frac{1}{p_{s} \rho}\right)
$$

where $\rho=\lambda / \mu$. As expected, the age approaches infinity as $p_{s}$ approaches zero.

Figure 11 shows the average peak age for a rate of 5 packets/second as a function of the packet loss rate. We note that the emulated age is similar to the theoretical age, but as 


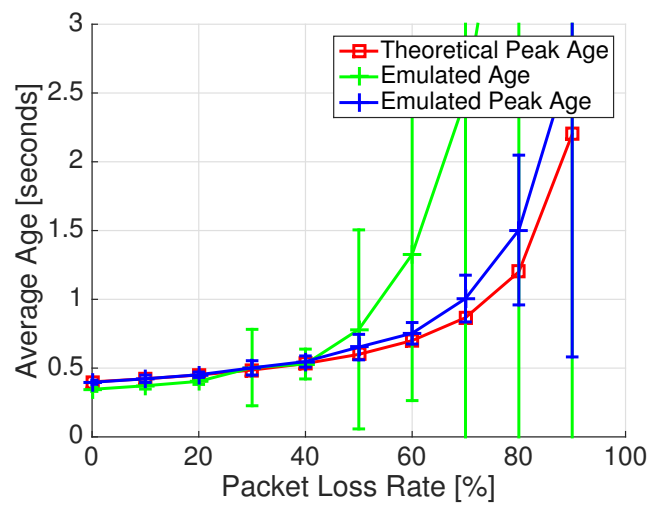

Fig. 11. Comparing theoretical and emulated average age for different packet loss $\%, 5$ packets/second rate.

packet loss gets very high, the variance in the sample set also gets very high. We also plot the emulated average age which is slightly less than the average peak age for small packet loss rates, but then grows much larger for higher packet loss rates. This can be interpreted as a high packet loss rate being less detrimental to the worst case age for an average packet than to the average age over all time.

We can explain this phenomenon from the mathematical expressions for average age and peak age, in which the average peak age weighs the interarrival time $X$ and system time $T$ equally, whereas the average age weighs $X$ more than $T$. In the error-free case, $X$ is less than $T$ on average in the regime we are interested in (i.e., the stable regime where queues are not yet saturated). However, as we increase the number of packet errors, the interarrival $X$ of successful packets increases while $T$ does not change. Therefore, the average age increases at a higher rate than the peak age.

\section{CONCLUSION AND FUtURE WORK}

In this work, we have investigated the real world performance of a new metric for the freshness of information in a network, known as the age of information. This metric directly describes the application layer performance for status updating systems, whereas traditional network metrics of throughput and delay are inadequate for optimizing the application's performance. We have studied the age metric in a realistic wireless system using network emulation tools CORE and
EMANE, and we have evaluated the age for a variety of different system settings and theoretical models, such as arrival and departure processes, average age and peak average age, number of flows, and packet loss. We also provide insight into the relationship between the age metric and metrics of interarrival time and latency. Additionally, we have derived some theoretical results for the average peak age metric and validated them with our emulation experiments.

It is important to validate the existing works on the age of information, which focus on theoretical, idealized system models. In our future work, we will further this effort by going beyond a single link to a more complex network setting, for which theoretical analysis is difficult, and emulation will be a valuable tool for understanding the age performance in networks.

\section{ACKNOWLEDGMENT}

This work was supported by the Office of Naval Research.

\section{REFERENCES}

[1] S. Kaul, M. Gruteser, V. Rai, and J. Kenney, "Minimizing age of information in vehicular networks," in IEEE Conference on Sensor, Mesh and Ad Hoc Communications and Networks (SECON), June 2011, pp. 350-358.

[2] S. Kaul, R. Yates, and M. Gruteser, "On piggybacking in vehicular networks," in IEEE Global Telecommunications Conference (GLOBECOM 2011), Dec 2011, pp. 1-5.

[3] _ - "Real-time status: How often should one update?" in Proc. IEEE INFOCOM, Orlando, FL, Mar. 2012, pp. 2731-2735.

[4] — "Status updates through queues," in Conference on Information Sciences and Systems (CISS), Princeton, NJ, Mar. 2012, pp. 1-6.

[5] R. D. Yates and S. Kaul, "Real-time status updating: Multiple sources," in Proc. IEEE International Symposium on Information Theory (ISIT), Cambridge, MA, Jul. 2012, pp. 2666-2670.

[6] M. Costa, M. Codreanu, and A. Ephremides, "Age of information with packet management," in IEEE International Symposium on Information Theory (ISIT), June 2014, pp. 1583-1587.

[7] C. Kam, S. Kompella, and A. Ephremides, "Age of information under random updates," in Proc. IEEE International Symposium on Information Theory (ISIT), Istanbul, Turkey, Jul. 2013, pp. 66-70.

[8] — "Effect of message transmission diversity on status age," in Information Theory (ISIT), 2014 IEEE International Symposium on, June 2014, pp. 2411-2415.

[9] (2015) Common Open Research Emulator (CORE) website. [Online] Available: http://www.nrl.navy.mil/itd/ncs/products/core

[10] (2015) Extendable Mobile Ad-hoc Network Emulator (EMANE) website. [Online]. Available: http://www.nrl.navy.mil/itd/ncs/products/ emane,https://github.com/adjacentlink/emane

[11] J. Ahrenholz, T. Goff, and B. Adamson, "Integration of the CORE and EMANE network emulators," in 2011 Military Communications Conference (MILCOM 2011), Nov 2011, pp. 1870-1875. 